\title{
EXISTENCE OF POSITIVE SOLUTION FOR KIRCHHOFF TYPE PROBLEM WITH CRITICAL DISCONTINUOUS NONLINEARITY
}

\author{
Giovany M. Figueiredo* and Gelson G. dos Santos \\ Universidade de Brasilia - UNB, Departamento de Matemática \\ CEP. 70910-900 , Brasília - DF, Brazil; giovany@unb.br \\ Universidade Federal do Pará - UFPA, Faculdade de Matemática \\ CEP. 6075-110, Belém - PA, Brazil; gelson@ymail.com
}

\begin{abstract}
In this paper we are concerned with existence of positive solution to the class of nonlinear problems of the Kirchhoff type given by

$$
\begin{aligned}
L_{\epsilon}(u) & =H(u-\beta) f(u)+u^{2^{*}-1} \quad \text { in } \mathbf{R}^{N}, \\
u & \in H^{1}\left(\mathbf{R}^{N}\right) \cap W^{2, \frac{q}{q-1}}\left(\mathbf{R}^{N}\right),
\end{aligned}
$$

where $N \geq 3, q \in\left(2,2^{*}\right), \epsilon, \beta>0$ are positive parameters, $f: \mathbf{R} \rightarrow \mathbf{R}$ is a continuous function, $H$ is the Heaviside function, i.e., $H(t)=0$ if $t \leq 0, H(t)=1$ if $t>0$ and

$$
L_{\epsilon}(u):=\left[M\left(\frac{1}{\epsilon^{N-2}} \int_{\mathbf{R}^{N}}|\nabla u|^{2} d x+\frac{1}{\epsilon^{N}} \int_{\mathbf{R}^{N}} V(x)|u|^{2} d x\right)\right]\left[-\epsilon^{2} \Delta u+V(x) u\right] .
$$

The function $M$ is a general continuous function. The function $V$ is a positive potential that satisfies following hypothesis: or $V$ satisfies the Palais-Smale condition or there is a bounded domain $\Omega$ in $\mathbf{R}^{N}$ such that $V$ has no critical point in $\partial \Omega$. Here we use a suitable truncation to apply a version of the penalization method of Del Pino and Felmer [16] combined with the Mountain Pass Theorem for locally Lipschitz functional.
\end{abstract}

\section{Introduction}

Problem of the type

$$
-\left(a+b \int_{\Omega}|\nabla u|^{2} d x\right) \Delta u=g(x, u) \quad \text { in } \Omega, \quad u=0 \text { on } \partial \Omega,
$$

where $\Omega$ is a bounded domain in $\mathbf{R}^{N}$ is very known as Kirchhoff problem. Its hyperbolic equation version which is given by

$$
\begin{cases}u_{t t}-\left(a+b \int_{\Omega}|\nabla u|^{2} d x\right) \Delta u=g(x, u) & \text { in } \Omega \times(0, T), \\ u=0 & \text { on } \partial \Omega \times(0, T), \\ u(x, 0)=u_{0}(x), u_{t}(x, 0)=u_{1}(x) & \text { in } \Omega,\end{cases}
$$

was studied in the first time by Kirchhoff in [26] with $N=1$. This equation extends the classical d'Alembert's wave equation by considering the effects of the changes in the length of the strings during the vibrations.

Many authors have studied problem (1) using Variational methods and exploring the compactness of $H_{0}^{1}(\Omega)$ in $L^{s}(\Omega)$ with $1 \leq s<2^{*}$. Another widely used situation is the dimension $N=3$. In general the dimension $N=3$ avoids the competition of the

https://doi.org/10.5186/aasfm.2019.4453

2010 Mathematics Subject Classification: Primary 35A15, 35B33, 35B25, 35J60.

Key words: Variational methods, critical exponents, Kirchhoff equation, discontinuous nonlinearity.

*Supported by CNPq, Capes and Fap-Df. 
term $\left(a+b \int_{\Omega}|\nabla u|^{2} d x\right)$ with the growth of nonlinearity. For more information on this competition see [20, Introduction]. Since it is impossible to cite all the authors that study problem (1) with $N=3$, we cite the most recent publications, such as [6], [15], [27], [29] and [31].

Problem (1) in $\mathbf{R}^{N}$ is more difficult due to the lack of compactness of Sobolev immersions. In general, in order to overcome this difficult, the author put a potential which the geometry allows to recover the compactness. See here the latest publications with these arguments, [11], [18], [24], [25], [32] and [33].

In this paper we are concerned with existence of positive solution to the class of nonlinear problems of the Kirchhoff type given by

$$
\left\{\begin{array}{l}
L_{\epsilon}(u)=H(u-\beta) f(u)+u^{2^{*}-1} \quad \text { in } \mathbf{R}^{N} \\
u \in H^{1}\left(\mathbf{R}^{N}\right) \cap W^{2, \frac{q}{q-1}}\left(\mathbf{R}^{N}\right)
\end{array}\right.
$$

where $N \geq 3, q \in\left(2,2^{*}\right), \epsilon, \beta>0$ are positive parameters, $f: \mathbf{R} \rightarrow \mathbf{R}$ is a continuous function, $H$ is the Heaviside function, i.e., $H(t)=0$ if $t \leq 0, H(t)=1$ if $t>0$ and

$$
L_{\epsilon}(u):=\left[M\left(\frac{1}{\epsilon^{N-2}} \int_{\mathbf{R}^{N}}|\nabla u|^{2} d x+\frac{1}{\epsilon^{N}} \int_{\mathbf{R}^{N}} V(x)|u|^{2} d x\right)\right]\left[-\epsilon^{2} \Delta u+V(x) u\right] .
$$

In order to enunciate the main result, we need to give some hypotheses on the functions $M, V$ and $f$. The hypothesis on the continuous function $M:[0,+\infty) \rightarrow$ $(0, \infty)$ is the following:

$\left(M_{1}\right)$ The function $M$ is nondecreasing.

Now we give some examples of functions that satisfy the hypothesis $\left(M_{1}\right)$. The example $M(t)=m_{0}+b t$ with $m_{0}, b>0$ was considered in [26]. Another examples are $M(t)=\log (t+1)+1$ or $M(t)=\exp t^{2}$.

The hypotheses on the continuous function $V: \mathbf{R}^{N} \rightarrow \mathbf{R}$ are the following:

$\left(V_{0}\right)$ There is $V_{0}>0$ such that $V(x) \geq V_{0}$, for all $x \in \mathbf{R}^{N}$.

$\left(V_{1}\right) V \in C^{2}\left(\mathbf{R}^{N}\right)$ and $V, \frac{\partial V}{\partial x_{i}}, \frac{\partial^{2} V}{\partial x_{i} \partial x_{j}}$ are bounded in $\mathbf{R}^{N}, i, j=1,2, \ldots, N$.

$\left(V_{2}\right) V$ verifies the Palais-Smale condition, that is, if $\left(x_{n}\right) \subset \mathbf{R}^{N}$ is such that, $\left(V\left(x_{n}\right)\right)$ is bounded and $\nabla V\left(x_{n}\right) \rightarrow 0$, then, $\left(x_{n}\right)$ possesses a convergent subsequence in $\mathbf{R}^{N}$.

$\left(V_{3}\right)$ There is a bounded domain $\Omega \subset \mathbf{R}^{N}$, such that $\nabla V(x) \neq 0$, for all $x \in \partial \Omega$.

The hypotheses on the continuous function $f: \mathbf{R} \rightarrow \mathbf{R}$ are the following:

$\left(f_{1}\right)$ For all $t \in \mathbf{R}$, there are $C>0$ and $q \in\left(2,2^{*}\right)$ such that

$$
|f(t)| \leq C\left(1+|t|^{q-1}\right) \text {. }
$$

$\left(f_{2}\right)$ For all $t \in \mathbf{R}$, there exists $\theta \in\left(2,2^{*}\right)$ such that

$$
0<\theta F(t)=\theta \int_{0}^{t} f(s) d s \leq t f(t) .
$$

$\left(f_{3}\right)$ There is $\beta>0$, that will be fixed later, such that

$$
H(t-2 \beta) \leq f(t), \quad \text { for all } t \in \mathbf{R} .
$$

$\left(f_{4}\right) \limsup _{t \rightarrow 0^{+}} \frac{f(t)}{t}=0$ and $f(t)=0$ if $t \leq 0$. 
A typical example of a function satisfying the conditions $\left(f_{1}\right),\left(f_{2}\right),\left(f_{3}\right)$ and $\left(f_{4}\right)$ is given by

$$
f(t)=\sum_{k=1}^{l} \frac{|t|^{q_{k}-1}}{2 \beta^{q_{k}-1}} \text { if } t \geq 0 \text { and } q_{k} \in\left(2,2^{*}\right) \text { and } f(t)=0 \text { if } t \leq 0 .
$$

Before our main result, we define weak solution and strong solution for problem (2). A weak solution for (2), we understand as a function $u_{\epsilon} \in H^{1}\left(\mathbf{R}^{N}\right) \cap W^{2, \frac{q}{q-1}}\left(\mathbf{R}^{N}\right)$ verifying

$$
L_{\epsilon}\left(u_{\epsilon}(x)\right)-u_{\epsilon}^{2^{*}-1}(x) \in\left[\underline{f}\left(u_{\epsilon}(x)\right), \bar{f}\left(u_{\epsilon}(x)\right)\right] \text { a.e. in } \mathbf{R}^{N},
$$

where $\underline{f}(t)$ and $\bar{f}(t)$ are $N$-measurable functions (see [9]) defined by

$$
\underline{f}(t):=\left.\lim _{\delta \downarrow 0} \operatorname{essinf}\right|_{|t-s|<\delta} H(s-\beta) f(s) \quad \text { and } \quad \bar{f}(t):=\lim _{\delta \downarrow 0} \operatorname{ess} \sup _{|t-s|<\delta} H(s-\beta) f(s) .
$$

By a strong solution for (2), we understand as a function $u_{\epsilon} \in H^{1}\left(\mathbf{R}^{N}\right) \cap$ $W^{2, \frac{q}{q-1}}\left(\mathbf{R}^{N}\right)$ verifying

$$
L_{\epsilon}\left(u_{\epsilon}(x)\right)=H\left(u_{\epsilon}(x)-\beta\right) f\left(u_{\epsilon}(x)\right)+u_{\epsilon}^{2^{*}-1}(x) \quad \text { a.e. in } \mathbf{R}^{N} .
$$

Our main result is as follows:

Theorem 1.1. Suppose that $M$ satisfies $\left(M_{1}\right), f$ satisfies $\left(f_{1}\right),\left(f_{2}\right),\left(f_{3}\right),\left(f_{4}\right)$ and $V$ satisfies $\left(V_{0}\right),\left(V_{1}\right)$ and $\left(V_{2}\right)$ or $\left(V_{0}\right),\left(V_{1}\right)$ and $\left(V_{3}\right)$. Then, there exists $\epsilon^{*}>0$ small, such that for each $\epsilon \in\left(0, \epsilon^{*}\right)$, the problem (2) has a weak positive solution $u_{\epsilon}$. Moreover:

(i) The set $\left\{x \in \mathbf{R}^{N}: u_{\epsilon}(x)=\beta\right\}$ has null measure and so $u_{\epsilon}$ satisfies (3).

(ii) The set $\left\{x \in \mathbf{R}^{N}: u_{\epsilon}(x)>\beta\right\}$ has positive measure.

Some physical problems are related to discontinuous surface

$$
\Gamma_{\beta}(u)=\{x \in \Omega ; u(x)=\beta\}
$$

which causes difficulties in analyzing this kind of problems. The interest in the study of nonlinear partial differential equations with discontinuous nonlinearities has increased because many free boundary problems arising in mathematical physics may be stated in this form. Among these problems, we have the obstacle problem, the seepage surface problem, and the Elenbaas equation, see for example [10, 8, 9]. Still related to elliptic problems with discontinuous nonlinearity, we cite the papers of Alves, Bertone and Gonçalves [2], Alves, Santos and Gonçalves [3], Badiale [4, 5], Dinu [17], Radulescu [30], Gazzola and Radulescu [21] and their references. Several techniques have been developed or applied in their study, such as variational methods for nondifferentiable functionals, lower and upper solutions, global branching, and the theory of multivalued mappings.

Our result is related with the articles [1], [14] and [19]. Alves in [1] used in the first time the hypotheses on the potential $V$ in the problem

$$
-\epsilon^{2} \Delta u+V(x) u=g(u) \text { in } \mathbf{R}^{N},
$$

where $g$ was continuous and subcritical. The version of this problem with discontinuous nonlinearity was studied in [19]. In [14] was studied a problem with a nonlocal operator and discontinuous nonlinearity in bounded domain. But in this paper, the nonlocal term does not contemplate the Kirchhoff function.

We have completed some previous results in the following sense: 
i) Here, we can not use Variational Methods for $C^{1}$ functionals, because in our case, the energy functional is only locally Lipschitz continuous, and so, we have to use Variational Methods for for nondifferentiable functionals, see Section 2 for more details.

ii) In [1] was necessary some estimates involving the boundedness of solution of an auxiliary problem (see Lemma 2.1 in [1]). Since our problema has a nonlocal term and the functional associated is only locally Lipschitz continuous it was necessary to develop new arguments to get the boundedness of solution of auxiliary problemas (see Lemma 3.6).

iii) This paper is a version of [19] considering the Kirchhoff term and critical growth.

iv) In this paper we consider $N \geq 3$. In order to overcome the competition between the operator and the nonlinearity, we make a truncation the operator like [20].

This paper is organized as follows. In the Section 2 we make a revision about functional $\operatorname{Lip}_{\text {loc }}$. In the Section 3 we make a truncation of the operator and another truncation in the nonlinearity. After that we show existence of positive solution of an auxiliary problem. Finally, we show that the solution of auxiliary problem is a solution of original problem in the Sections 4 and 5 .

\section{Variational framework and preliminary results}

In this section, for the reader's convenience, we recall some definitions and basic results on the critical point theory of locally Lipschitz continuous functionals as developed by Chang [10], Clarke [12, 13] and Grossinho and Tersian [23].

Let $X$ be a real Banach space. A functional $I: X \rightarrow \mathbf{R}$ is locally Lipschitz continuous, $I \in \operatorname{Lip}_{\text {loc }}(X, \mathbf{R})$ for short, if given $u \in X$ there is an open neighborhood $V:=V_{u} \subset X$ and some constant $K=K_{V}>0$ such that

$$
\left|I\left(v_{2}\right)-I\left(v_{1}\right)\right| \leq K\left\|v_{2}-v_{1}\right\|, \quad v_{i} \in V, \quad i=1,2 .
$$

The directional derivative of $I$ at $u$ in the direction of $v \in X$ is defined by

$$
I^{0}(u ; v)=\limsup _{h \rightarrow 0} \frac{I(u+h+\sigma v)-I(u+h)}{\sigma} .
$$

Hence $I^{0}(u ;$.$) is continuous, convex and its subdifferential at z \in X$ is given by

$$
\Upsilon^{0}(u ; z)=\left\{\mu \in X^{*} ; I^{0}(u ; v) \geq I^{0}(u ; z)+\langle\mu, v-z\rangle, v \in X\right\},
$$

where $\langle.,$.$\rangle is the duality pairing between X^{*}$ and $X$. The generalized gradient of $I$ at $u$ is the set

$$
\partial I(u)=\left\{\mu \in X^{*} ;\langle\mu, v\rangle \leq I^{0}(u ; v), v \in X\right\} .
$$

Since $I^{0}(u ; 0)=0, \partial I(u)$ is the subdifferential of $I^{0}(u ; 0)$. A few definitions and properties will be recalled below.

$$
\partial I(u) \subset X^{*} \text { is convex, non-empty and weak*-compact, }
$$

$$
\Lambda(u)=\min \left\{\|\mu\|_{X^{*}} ; \mu \in \partial I(u)\right\}
$$

and

$$
\partial I(u)=\left\{I^{\prime}(u)\right\} \text { if } I \in C^{1}(X, \mathbf{R}) .
$$

A critical point of $I$ is an element $u_{0} \in X$ such that $0 \in \partial I\left(u_{0}\right)$ and a critical value of $I$ is a real number $c$ such that $I\left(u_{0}\right)=c$ for some critical point $u_{0} \in X$. 
We say that $I \in \operatorname{Lip}_{\text {loc }}(X, \mathbf{R})$ satisfies the Palais-Smale condition at level $c \in \mathbf{R}$ $\left((P S)_{c}\right.$-condition for short $)$, if the following holds: Every sequence $\left(u_{n}\right) \subset X$, such that $I\left(u_{n}\right) \rightarrow c$ and $\Lambda\left(u_{n}\right) \rightarrow 0$ has a strongly convergent subsequence.

Theorem 2.1. Let $X$ be a Banach space and let $I \in \operatorname{Lip}_{\text {loc }}(X, \mathbf{R})$ with $I(0)=0$. Suppose there are numbers $\varrho, r>0$ and $e \in X$, such that

(i) $I(u) \geq \varrho$, for all $u \in X ;\|u\|=r$,

(ii) $I(e)<0$ and $\|e\|>r$.

Let

$$
c=\inf _{\gamma \in \Gamma} \max _{t \in[0,1]} I(\gamma(t)) \quad \text { and } \quad \Gamma=\{\gamma \in C([0,1], X): \gamma(0)=0 \text { and } \gamma(1)=e\} .
$$

Then $c \geq \varrho$ and there is a sequence $\left(u_{n}\right) \subset X$ (named a $(P S)_{c}$-sequence) satisfying

$$
I\left(u_{n}\right) \rightarrow c \text { and } \Lambda\left(u_{n}\right) \rightarrow 0 .
$$

If, in addition, $I$ satisfies the $(P S)_{c}$-condition, then $c$ is a critical value of $I$.

\section{An auxiliary problem}

Using the change variable $v(x)=u(\epsilon x)$, it is possible to prove that (2) is equivalent to the following problem

$$
\begin{aligned}
\widetilde{L}_{\epsilon}(u) & =H(u-\beta) f(u)+u^{2^{*}-1} \quad \text { in } \mathbf{R}^{N}, \\
u & \in H^{1}\left(\mathbf{R}^{N}\right) \cap W^{2, \frac{q}{q-1}}\left(\mathbf{R}^{N}\right)
\end{aligned}
$$

where

$$
\widetilde{L}_{\epsilon}(u):=\left[M\left(\int_{\mathbf{R}^{N}}|\nabla u|^{2}+\int_{\mathbf{R}^{N}} V(\epsilon x)|u|^{2}\right)\right][-\Delta u+V(\epsilon x) u] .
$$

In this section, we fix some notations and an auxiliary problem, which are key points in our arguments. To establish the existence of positive solutions, we will make a truncation on the function $M$ as in [20] and we will adapt the penalization method due to Del Pino and Felmer [16], which consists in considering an auxiliary problem. To this end, we need to fix some notations.

In this section, on the function $\mathrm{V}$, we assume only $\left(V_{0}\right)$ and $\left(V_{1}\right)$. From $\left(M_{1}\right)$, there exists $t_{0}>0$ such that $M(0) \leq M\left(t_{0}\right)<\frac{\theta}{2} M(0)$ where $\theta$ is given by $\left(f_{2}\right)$. We set

$$
M_{0}(t):= \begin{cases}M(t), & \text { if } 0 \leq t \leq t_{0} \\ M\left(t_{0}\right), & \text { if } t \geq t_{0}\end{cases}
$$

Using $\left(M_{1}\right)$ and $(7)$, we get

$$
M(0) \leq M_{0}(t)<\frac{\theta}{2} M(0), \quad \text { for all } t \geq 0 .
$$

Note that by $\left(f_{4}\right)$ we have

$$
\lim _{t \rightarrow 0}\left[\frac{f(t)}{t}+t^{2^{*}-2}\right]=0 \text { and } \lim _{t \rightarrow+\infty}\left[\frac{f(t)}{t}+t^{2^{*}-2}\right]=+\infty .
$$

Then there exists $a>0$ such that

$$
\frac{f(a)}{a}+a^{2^{*}-2}=\frac{V_{0}}{k}
$$


where $V_{0}$ is given in $\left(V_{0}\right)$ and

$$
k>\frac{2 \theta}{\theta M(0)-2 M\left(t_{0}\right)},
$$

where $\theta$ is given in $\left(f_{2}\right)$. In $\left(f_{3}\right)$ we choose $\beta>0$ such that $\beta<a<2 \beta$.

Using the above numbers, let us define the function

$$
\widetilde{f}(t)= \begin{cases}0 & \text { if } t \leq 0, \\ H(t-\beta) f(t)+t^{2^{*}-1} & \text { if } 0<t<a, \\ \frac{V_{0}}{k} t & \text { if } t \geq a .\end{cases}
$$

Fixing $\Omega \subset \mathbf{R}^{N}$ a bounded domain and using the function $\tilde{f}$, let us consider the function

$$
g(x, t)=\chi_{\Omega}(x)\left(H(t-\beta) f(t)+t^{2^{*}-1}\right)+\left(1-\chi_{\Omega}(x)\right) \tilde{f}(t),
$$

where $\chi_{\Omega}$ is the characteristic function related to $\Omega$. Now consider the auxiliary problem

$$
\left\{\begin{array}{l}
M_{0}\left(\int_{\mathbf{R}^{N}}\left(|\nabla u|^{2}+V(\epsilon x)|u|^{2}\right)\right)(-\Delta u+V(\epsilon x) u)=g(\epsilon x, u), \\
u \in H^{1}\left(\mathbf{R}^{N}\right) \cap W^{2, \frac{q}{q-1}}\left(\mathbf{R}^{N}\right) .
\end{array}\right.
$$

A weak solution for $\left(P_{\epsilon}\right)_{a}$, we understand as a function $u_{\epsilon} \in H^{1}\left(\mathbf{R}^{N}\right) \cap W^{2, \frac{q}{q-1}}\left(\mathbf{R}^{N}\right)$ verifying

$$
\begin{aligned}
& M_{0}\left(\int_{\mathbf{R}^{N}}\left(\left|\nabla u_{\epsilon}\right|^{2}+V(\epsilon x)\left|u_{\epsilon}\right|^{2}\right)\right)\left(-\Delta u_{\epsilon}(x)+V(\epsilon x) u_{\epsilon}(x)\right) \\
& \in\left[\underline{g}\left(\epsilon x, u_{\epsilon}(x)\right), \bar{g}\left(\epsilon x, u_{\epsilon}(x)\right)\right] \quad \text { a.e. in } \quad \mathbf{R}^{N},
\end{aligned}
$$

where

$$
\underline{g}(x, t):=\lim _{\delta \downarrow 0} \operatorname{ess} \inf _{|t-s|<\delta} g(x, s) \quad \text { and } \quad \bar{g}(x, t):=\lim _{\delta \downarrow 0} \operatorname{ess} \sup _{|t-s|<\delta} g(x, s),
$$

which are $N$-measurable.

By a strong solution for $\left(P_{\epsilon}\right)_{a}$, we understand as a function $u_{\epsilon} \in H^{1}\left(\mathbf{R}^{N}\right) \cap$ $W^{2, \frac{q}{q-1}}\left(\mathbf{R}^{N}\right)$ verifying

$M_{0}\left(\int_{\mathbf{R}^{N}}\left(\left|\nabla u_{\epsilon}\right|^{2}+V(\epsilon x)\left|u_{\epsilon}\right|^{2}\right)\right)\left(-\Delta u_{\epsilon}(x)+V(\epsilon x) u_{\epsilon}(x)\right)=g\left(\epsilon x, u_{\epsilon}(x)\right)$ a.e. in $\mathbf{R}^{N}$.

Remark 3.1. The problem $\left(P_{\epsilon}\right)_{a}$ is strongly related to (6), because if $u_{\epsilon}$ is a solution of $\left(P_{\epsilon}\right)_{a}$ verifying $\left\|u_{\epsilon}\right\| \leq t_{0}$ and $u_{\epsilon}(x) \leq a$ in $\mathbf{R}^{N} \backslash \Omega_{\epsilon}$ where $\Omega_{\epsilon}=\Omega / \epsilon$, then by definitions of $M_{0}$ and $g, u_{\epsilon}$ will be a solution for (6).

From $\left(f_{1}\right)-\left(f_{4}\right)$, the function $g$ satisfies the following conditions uniformly in $x \in \mathbf{R}^{N}$ :

$\left(g_{1}\right) g(x, t)=0$ for all $t \leq 0$ and $\lim \sup _{|t| \rightarrow 0} \frac{g(x, t)}{|t|}=0$.

$\left(g_{2}\right) g(x, t)=H(t-\beta) f(t)+t^{2^{*}-1}$, for all $x \in \Omega, t>0$, or $x \in \Omega^{c}$ and $t \in[0, a]$.

$\left(g_{3}\right) g(x, t) \leq f(t)+t^{2^{*}-1}$ for all $x \in \mathbf{R}^{N}, t \in \mathbf{R}$.

$\left(g_{4}\right) 0<\theta G(x, t)=\theta \int_{0}^{t} g(x, s) d s \leq \underline{g}(x, t) t$, for all $x \in \Omega, t>0$, and $0<$ $2 G(x, t) \leq \underline{g}(x, t) t \leq \bar{g}(x, t) t \leq \frac{1}{k} V_{0} \bar{t}^{2}$, for all $x \notin \Omega, t \geq 0$.

We note that from $\left(V_{0}\right)$ and $\left(V_{1}\right)$, we can work in $H^{1}\left(\mathbf{R}^{N}\right)$ with the norm

$$
\|u\|:=\left(\int_{\mathbf{R}^{N}}|\nabla u|^{2}+V(\epsilon x)|u|^{2}\right)^{1 / 2}, \quad u \in H^{1}\left(\mathbf{R}^{N}\right),
$$


Existence of positive solution for Kirchhoff type problem with critical discontinuous nonlinearity 993

which is equivalent to the usual norm.

Let $\widehat{\Psi}_{\epsilon}$ be a functional given

$$
\widehat{\Psi}_{\epsilon}(u)=\int_{\mathbf{R}^{N}} G(\epsilon x, u), \quad \text { for all } u \in L^{2^{*}}\left(\mathbf{R}^{N}\right) .
$$

Using $\left(g_{2}\right),\left(f_{1}\right)$ and $(11)$, we obtain a constant $C=C(\beta)>0$, such that

$$
|g(\epsilon x, t)| \leq C|t|^{2^{*}-1}, \quad \text { for all } x \in \mathbf{R}^{N}, t \in \mathbf{R} .
$$

As an immediate consequence of (12), we obtain the following version of Theorems 2.1 and 2.2 due to Chang [10] and its proof will be omitted.

Lemma 3.2. The functional $\widehat{\Psi}_{\epsilon}$ satisfies:

(i) $\widehat{\Psi}_{\epsilon} \in \operatorname{Lip}_{\mathrm{loc}}\left(L^{2^{*}}\left(\mathbf{R}^{N}\right), \mathbf{R}\right)$ and

$$
\partial \widehat{\Psi}_{\epsilon}(u) \subseteq \int_{\mathbf{R}^{N}} \partial G(\epsilon x, u), \quad \text { for all } u \in L^{2^{*}}\left(\mathbf{R}^{N}\right),
$$

in the sense that for every $\xi^{*} \in \partial \widehat{\Psi}_{\epsilon}(u)$ there exists $\xi \in L^{\frac{2^{*}}{2^{*}-1}}\left(\mathbf{R}^{N}\right)$, such that, $\xi(x) \in \partial G(\epsilon x, u(x)) \subset[\underline{g}(\epsilon x, u(x)), \bar{g}(\epsilon x, u(x))]$ a.e. in $\mathbf{R}^{N}$ and

$$
\left\langle\xi^{*}, \varphi\right\rangle=\int_{\mathbf{R}^{N}} \xi \varphi, \quad \text { for all } \varphi \in L^{2^{*}}\left(\mathbf{R}^{N}\right) .
$$

(ii) If $\Psi_{\epsilon}=\left.\widehat{\Psi}_{\epsilon}\right|_{H^{1}\left(\mathbf{R}^{N}\right)}$, then $\partial \Psi_{\epsilon}(u)=\partial \widehat{\Psi}_{\epsilon}(u)$, for all $u \in H^{1}\left(\mathbf{R}^{N}\right)$.

The functional associated with $\left(P_{\epsilon}\right)_{a}$ is given by $J_{\epsilon}(u)=Q_{\epsilon}(u)-\Psi_{\epsilon}(u), u \in$ $H^{1}\left(\mathbf{R}^{N}\right)$, where

$$
Q_{\epsilon}(u)=\frac{1}{2} \widehat{M}_{0}\left(\|u\|^{2}\right), \quad \widehat{M}_{0}(t)=\int_{0}^{t} M_{0}(s) d s \quad \text { and } \quad \Psi_{\epsilon}(u)=\int_{\mathbf{R}^{N}} G(\epsilon x, u) .
$$

The functional $Q_{\epsilon} \in C^{1}\left(H^{1}\left(\mathbf{R}^{N}\right), \mathbf{R}\right), J_{\epsilon} \in \operatorname{Lip}_{\text {loc }}\left(H^{1}\left(\mathbf{R}^{N}\right), \mathbf{R}\right)$ with

$$
\left\langle Q_{\epsilon}^{\prime}(u), \varphi\right\rangle=M_{0}\left(\|u\|^{2}\right) \int_{\mathbf{R}^{N}}(\nabla u \nabla \varphi+V(\epsilon x) u \varphi), \quad \text { for all } u, \varphi \in H^{1}\left(\mathbf{R}^{N}\right)
$$

and

$$
\partial J_{\epsilon}(u)=\left\{Q_{\epsilon}^{\prime}(u)\right\}-\partial \Psi_{\epsilon}(u), \quad \text { for all } u \in H^{1}\left(\mathbf{R}^{N}\right) .
$$

Lemma 3.3. Let $\left(u_{n}\right)$ be a $(P S)_{c}$ sequence for $J_{\epsilon}$, then $\left(u_{n}\right)$ is bounded in $H^{1}\left(\mathbf{R}^{N}\right)$.

Proof. Let $\left(w_{n}\right) \subset H^{-1}\left(\mathbf{R}^{N}\right)$ be such that $\Lambda\left(u_{n}\right)=\left\|w_{n}\right\|_{*}=o_{n}(1)$, where $\Lambda$ appeared in (5). Since $w_{n} \in \partial J_{\epsilon}\left(u_{n}\right)$, there exists $\xi_{n}^{*} \in \partial \Psi_{\epsilon}\left(u_{n}\right)$ verifying

$$
\left\langle w_{n}, \varphi\right\rangle=\left\langle Q_{\epsilon}^{\prime}\left(u_{n}\right), \varphi\right\rangle-\left\langle\xi_{n}^{*}, \varphi\right\rangle, \quad \text { for all } \varphi \in H^{1}\left(\mathbf{R}^{N}\right) .
$$

From (13), $\left(M_{1}\right)$ and $\left(g_{4}\right)$, we have

$$
J_{\epsilon}\left(u_{n}\right)-\frac{1}{\theta}\left\langle w_{n}, u_{n}\right\rangle \geq\left(\frac{M(0)}{2}-\frac{M\left(t_{0}\right)}{\theta}\right)\left\|u_{n}\right\|^{2}+\int_{\Omega_{\epsilon}^{c}}\left[\frac{1}{\theta} \xi_{n} u_{n}-G\left(\epsilon x, u_{n}\right)\right],
$$

where $\xi_{n} \in\left[\underline{g}\left(\epsilon x, u_{n}\right), \bar{g}\left(\epsilon x, u_{n}\right)\right]$.

Since $\theta>2$, the relation $\left(g_{4}\right)$ imply that

$$
\int_{\Omega_{\epsilon}^{c}}\left[\frac{1}{\theta} \xi_{n} u_{n}-G\left(\epsilon x, u_{n}\right)\right] \geq \frac{2-\theta}{\theta} \frac{1}{2 k} \int_{\Omega_{\epsilon}^{c}} V(\epsilon x)\left|u_{n}\right|^{2} .
$$


Using (14) and (15), we obtain

$$
C+C\left\|u_{n}\right\| \geq\left(\frac{M(0)}{2}-\frac{M\left(t_{0}\right)}{\theta}\right)\left\|u_{n}\right\|^{2}+\frac{2-\theta}{\theta} \frac{1}{2 k}\left\|u_{n}\right\|^{2} .
$$

By choice of $k$, see (10), we have,

$$
C+C\left\|u_{n}\right\| \geq \frac{1}{2}\left(\frac{M(0)}{2}-\frac{M\left(t_{0}\right)}{\theta}\right)\left\|u_{n}\right\|^{2} .
$$

Therefore, $\left(u_{n}\right)$ is bounded in $H^{1}\left(\mathbf{R}^{N}\right)$.

Lemma 3.4. Let $\left(u_{n}\right)$ be a $(P S)_{c}$ sequence for $J_{\epsilon}$. Then for each $\delta>0$, there exists $R=R(\delta)>0$, such that

$$
\limsup _{n \rightarrow \infty} \int_{\mathbf{R}^{N} \backslash B_{R}(0)}\left[\left|\nabla u_{n}\right|^{2}+V(\epsilon x)\left|u_{n}\right|^{2}\right]<\delta .
$$

Proof. Let $\left(w_{n}\right), \xi_{n}^{*}$ and $\xi_{n}$ used in the proof of the previous lemma and $\eta_{R} \in$ $C^{\infty}\left(\mathbf{R}^{N},[0,1]\right)$, such that $\eta_{R}(x)=0$ in $B_{R}(0), \eta_{R}(x)=1$ in $B_{2 R}(0)^{c}$ and $\left|\nabla \eta_{R}(x)\right| \leq$ $C / R$ in $\mathbf{R}^{N}$, where $C>0$ is a constant independent on $R$.

By Lemma 3.3, $\left(u_{n}\right)$ is bounded in $H^{1}\left(\mathbf{R}^{N}\right)$ and $\left\langle w_{n}, \eta_{R} u_{n}\right\rangle=o_{n}(1)$. From (13), we obtain

$M(0) \int_{\mathbf{R}^{N}} \eta_{R}\left[\left|\nabla u_{n}\right|^{2}+V(\epsilon x)\left|u_{n}\right|^{2}\right] \leq \int_{\mathbf{R}^{N}} \xi_{n} \eta_{R} u_{n}-M_{0}\left(\left\|u_{n}\right\|^{2}\right) \int_{\mathbf{R}^{N}} u_{n} \nabla \eta_{R} \nabla u_{n}+o_{n}(1)$.

Fixed $R>0$, such that $\Omega_{\epsilon} \subset B_{\frac{R}{2}}(0)$. Since $\xi_{n} \in\left[\underline{g}\left(\epsilon x, u_{n}\right), \bar{g}\left(\epsilon x, u_{n}\right)\right]$, by $\left(g_{4}\right)$,

$$
\int_{\mathbf{R}^{N}} \xi_{n} \eta_{R} u_{n} \leq \frac{1}{k} \int_{\mathbf{R}^{N}} \eta_{R} V(\epsilon x)\left|u_{n}\right|^{2}
$$

combining this with (8), we find

$$
\left(M(0)-\frac{1}{k}\right) \int_{\mathbf{R}^{N}} \eta_{R}\left[\left|\nabla u_{n}\right|^{2}+V(\epsilon x)\left|u_{n}\right|^{2}\right] \leq M(0) \frac{\theta}{2} \frac{C}{R}\left|u_{n}\right|_{L^{2}}\left|\nabla u_{n}\right|_{L^{2}}+o_{n}(1) .
$$

Since $k>\frac{1}{M(0)}$, see (10), we conclude the proof.

Let $S$ be the best constant for Sobolev's embedding $D^{1,2}\left(\mathbf{R}^{N}\right) \hookrightarrow L^{2^{*}}\left(\mathbf{R}^{N}\right)$.

Proposition 3.5. The functional $J_{\epsilon}$ verifies the $(P S)_{c}$ condition in $H^{1}\left(\mathbf{R}^{N}\right)$ for $c<\left(\frac{M(0)}{2}-\frac{M\left(t_{0}\right)}{\theta}\right)\left(M(0) S^{\frac{N}{N-2}}\right)^{\frac{N-2}{2}}$.

Proof. By Lemma 3.3, $\left(u_{n}\right)$ is bounded in $H^{1}\left(\mathbf{R}^{N}\right)$. Taking a subsequence, we may assume that $u_{n} \rightarrow u$ in $H^{1}\left(\mathbf{R}^{N}\right), u_{n}(x) \rightarrow u(x)$ a.e. in $\mathbf{R}^{N}$,

$$
\left|\nabla u_{n}\right|^{2} \rightarrow|\nabla u|^{2}+\mu \quad \text { and }\left|u_{n}\right|^{2^{*}} \rightarrow|u|^{2^{*}}+\nu \quad \text { (weak*-sense of measure). }
$$

Using the concentration compactness-principle by Lions [26, Lemma 2.1], we obtain at most countable index set $\Sigma$, sequences $\left(x_{i}\right) \subset \mathbf{R}^{N},\left(\mu_{i}\right),\left(\nu_{i}\right) \subset[0, \infty)$, such that

$$
\nu=\sum_{i \in \Sigma} \nu_{i} \delta x_{i}, \quad \mu \geq \sum_{i \in \Sigma} \mu_{i} \delta x_{i} \quad \text { and } \quad S \nu_{i}^{\frac{2}{2^{*}}} \leq \mu_{i}
$$

for all $i \in \Sigma$, where $\delta_{x i}$ is the Dirac mass at $x_{i}$.

Now we claim that $\Sigma=\emptyset$. Arguing by contradiction, assume that $\Sigma \neq \emptyset$ and fix $i \in \Sigma$. Let $w_{n}, \xi_{n}^{*}$ and $\xi_{n}$ used in the proof of the Lemma 3.3. 
Existence of positive solution for Kirchhoff type problem with critical discontinuous nonlinearity 995

Consider $\varphi \in C_{0}^{\infty}\left(\mathbf{R}^{N},[0,1]\right)$, such that, $\varphi \equiv 1$ in $B_{1}(0), \varphi \equiv 0$ in $\mathbf{R}^{N} \backslash B_{2}(0)$ and $|\nabla \varphi|_{L^{\infty}} \leq 2$. Defining $\varphi_{\varrho}(x)=\varphi\left(\frac{x-x_{i}}{\varrho}\right)$, where $\varrho>0$, we have that $\left(\varphi_{\varrho} u_{n}\right)$ is bounded in $H^{1}\left(\mathbf{R}^{N}\right)$, that implies

$$
M_{0}\left(\left\|u_{n}\right\|^{2}\right) \int_{\mathbf{R}^{N}}\left(\nabla u_{n} \nabla\left(\varphi_{\varrho} u_{n}\right)+V(\epsilon x) \varphi_{\varrho} u_{n}^{2}\right)=\int_{\Omega_{\epsilon}} \xi_{n} \varphi_{\varrho} u_{n}+\int_{\Omega_{\epsilon}^{c}} \xi_{n} \varphi_{\varrho} u_{n}+o_{n}(1) .
$$

Using $\left(M_{1}\right),\left(g_{3}\right)$ and $\left(g_{4}\right)$, we obtain

$$
\begin{aligned}
M(0) \int_{\mathbf{R}^{N}} \varphi_{\varrho}\left|\nabla u_{n}\right|^{2} \leq & -M_{0}\left(\left\|u_{n}\right\|^{2}\right) \int_{\mathbf{R}^{N}}\left(u_{n} \nabla u_{n} \nabla \varphi_{\varrho}+V(\epsilon x) \varphi_{\varrho} u_{n}^{2}\right) \\
& +C \int_{\mathbf{R}^{N}}\left|u_{n}\right|^{q} \varphi_{\varrho}+\int_{\mathbf{R}^{N}}\left|u_{n}\right|^{2^{*}} \varphi_{\varrho}+C \int_{\mathbf{R}^{N}}\left|u_{n}\right|^{2} \varphi_{\varrho} .
\end{aligned}
$$

Since $\left(u_{n}\right)$ is bounded in $H^{1}\left(\mathbf{R}^{N}\right)$, the support of $\varphi_{\varrho}$ is contained in $B_{2 \varrho}\left(x_{i}\right)$,

$$
\begin{aligned}
& \lim _{\varrho \rightarrow 0} \lim _{n \rightarrow \infty} M_{0}\left(\left\|u_{n}\right\|^{2}\right) \int_{\mathbf{R}^{N}}\left(u_{n} \nabla u_{n} \nabla \varphi_{\varrho}+V(\epsilon x) \varphi_{\varrho} u_{n}^{2}\right)=0, \\
& \lim _{\varrho \rightarrow 0} \lim _{n \rightarrow \infty} \int_{\mathbf{R}^{N}}\left|u_{n}\right|^{2} \varphi_{\varrho}=0 \text { and } \lim _{\varrho \rightarrow 0} \lim _{n \rightarrow \infty} \int_{\mathbf{R}^{N}}\left|u_{n}\right|^{q} \varphi_{\varrho} u_{n}=0 .
\end{aligned}
$$

Thus, by (17)

$$
M(0) \int_{\mathbf{R}^{N}} \varphi_{\varrho} d \mu \leq \int_{\mathbf{R}^{N}} \varphi_{\varrho} d \nu+o_{\varrho}(1) .
$$

Letting $\varrho \rightarrow 0$ and using standard theory of Radon measures, we conclude that $M(0) \mu_{i} \leq \nu_{i}$. From $(16), \mu_{i} \geq\left(M(0) S^{\frac{N}{N-2}}\right)^{\frac{N-2}{2}}$.

Once that $\left(u_{n}\right)$ is a sequence $(P S)_{c}$, arguing as in the proof of Lemma 3.3,

$$
\begin{aligned}
c & =J_{\epsilon}\left(u_{n}\right)-\frac{1}{\theta}\left\langle w_{n}, u_{n}\right\rangle+o_{n}(1) \\
& \geq\left(\frac{M(0)}{2}-\frac{M\left(t_{0}\right)}{\theta}\right) \int_{\mathbf{R}^{N}}\left|\nabla u_{n}\right|^{2} \varphi_{\varrho}+C_{0} \int_{\mathbf{R}^{N}} V(\epsilon x)\left|u_{n}\right|^{2} \varphi_{\varrho}+o_{n}(1) .
\end{aligned}
$$

Thus, we conclude that $c \geq\left(\frac{M(0)}{2}-\frac{M\left(t_{0}\right)}{\theta}\right)\left(M(0) S^{\frac{N}{N-2}}\right)^{\frac{N-2}{2}}$, which is a contradiction. Therefore, $\Sigma$ is empty and it follows that

$$
u_{n} \rightarrow u \text { in } L_{\mathrm{loc}}^{2^{*}}\left(\mathbf{R}^{N}\right)
$$

Recalling that

$$
M_{0}\left(\left\|u_{n}\right\|^{2}\right)\left\|u_{n}\right\|^{2}=\int_{\mathbf{R}^{N}} \xi_{n} u_{n}+o_{n}(1)
$$

and since $\xi_{n} \in\left[\underline{g}\left(\epsilon x, u_{n}\right), \bar{g}\left(\epsilon x, u_{n}\right)\right]$, by (12) and the boundedness of $\left(u_{n}\right)$ in $H^{1}\left(\mathbf{R}^{N}\right)$, we obtain that $\left(\xi_{n}\right)$ is bounded in $L^{\frac{2^{*}}{2^{*}-1}}\left(\mathbf{R}^{N}\right)$. Thus, up to a subsequence,

$$
\left\|u_{n}\right\| \rightarrow \sigma \text { in } \mathbf{R}, \quad \xi_{n} \rightarrow \xi \text { in } L^{\frac{2^{*}}{2^{*}-1}}\left(\mathbf{R}^{N}\right) \text { and } u_{n} \rightarrow u \text { in } H^{1}\left(\mathbf{R}^{N}\right) .
$$

From (13),

$$
M_{0}\left(\sigma^{2}\right)\|u\|^{2}=\int_{\mathbf{R}^{N}} \xi u
$$

Since

$$
\left|\int_{B_{R}(0)} \xi_{n} u_{n}-\int_{B_{R}(0)} \xi u\right| \leq\left|u_{n}-u\right|_{L^{2^{*}}\left(B_{R}(0)\right)}\left|\xi_{n}\right|_{L^{\frac{2^{*}}{2^{*}-1}\left(\mathbf{R}^{N}\right)}}+\left|\int_{B_{R}(0)}\left(\xi_{n}-\xi\right) u\right|,
$$


using (18), (20) and the Riesz representation theorem, we have

$$
\int_{B_{R}(0)} \xi_{n} u_{n} \rightarrow \int_{B_{R}(0)} \xi u
$$

The Lemma 3.4 implies that

$$
\limsup _{n \rightarrow \infty}\left|\int_{B_{R}^{c}(0)} \xi_{n} u_{n}\right|=o_{R}(1)
$$

Noting that

$$
\left|\int_{\mathbf{R}^{N}} \xi_{n} u_{n}-\int_{\mathbf{R}^{N}} \xi u\right| \leq\left|\int_{B_{R}(0)} \xi_{n} u_{n}-\int_{B_{R}(0)} \xi u\right|+\left|\int_{B_{R}^{c}(0)} \xi_{n} u_{n}\right|+o_{R}(1),
$$

using (22) and (23), we conclude that

$$
\int_{\mathbf{R}^{N}} \xi_{n} u_{n} \rightarrow \int_{\mathbf{R}^{N}} \xi u
$$

Combining (19), (21) and (24), we obtain $M\left(\left\|u_{n}\right\|^{2}\right)\left\|u_{n}\right\|^{2}=M\left(\sigma^{2}\right)\|u\|^{2}+o_{n}(1)$. Therefore, $u_{n} \rightarrow u$ in $H^{1}\left(\mathbf{R}^{N}\right)$.

Lemma 3.6. Let $\left(u_{n}\right)$ be a $(P S)_{c}$ sequence for $J_{\epsilon, \beta}$ with $c<\frac{1}{2}\left(\frac{M(0)}{2}-\frac{M\left(t_{0}\right)}{\theta}\right) t_{0}^{2}$. Then, there exists $n_{0}>0$, such that

$$
\left\|u_{n}\right\| \leq t_{0}, \quad \text { for all } n \geq n_{0},
$$

where $t_{0}$ is given by $(7)$.

Proof. Suppose, that the lemma is false, then we obtain a subsequence of $\left(u_{n}\right)$, that we are still denoting by $\left(u_{n}\right)$, that satisfies $\left\|u_{n}\right\|>t_{0}$, for all $n \in \mathbf{N}$. Using $w_{n} \in \partial J\left(u_{n}\right)$, we get

$$
c+o_{n}(1)=J_{\epsilon}\left(u_{n}\right)-\frac{1}{\theta}\left\langle w_{n}, u_{n}\right\rangle \geq \frac{1}{2}\left(\frac{M(0)}{2}-\frac{M\left(t_{0}\right)}{\theta}\right)\left\|u_{n}\right\|^{2} .
$$

Since $M\left(t_{0}\right)<\frac{\theta}{2} M(0)$, we have

$$
c \geq \frac{1}{2}\left(\frac{M(0)}{2}-\frac{M\left(t_{0}\right)}{\theta}\right) t_{0}^{2},
$$

which is a contradiction.

The number

$$
c^{*}:=\min \left\{t_{0}^{2},\left(M(0) S^{\frac{N}{N-2}}\right)^{\frac{N-2}{2}}\right\} \frac{1}{2}\left(\frac{M(0)}{2}-\frac{M\left(t_{0}\right)}{\theta}\right)
$$

is important in order to prove that the functional satisfies the $(P S)_{c}$ condition and that a solution of the auxiliary problem $\left(P_{\epsilon}\right)_{a}$ is a solution of the original problem (6).

In the next result we choose the constant $\beta$ of the condition $\left(f_{3}\right)$.

Lemma 3.7. Suppose that $\left(f_{1}\right),\left(f_{2}\right),\left(f_{3}\right),\left(f_{4}\right),\left(V_{0}\right),\left(V_{1}\right)$ are satisfied. Fixed $\epsilon^{*}, \beta>0$ small, for each $\epsilon \in\left(0, \epsilon^{*}\right)$, there are $\gamma_{0}>0$ and $v_{0} \in H^{1}\left(\mathbf{R}^{N}\right)$, which are independent of $\epsilon^{*}$ and $\beta$, such that

(i) $\max _{t \in\left[0, \gamma_{0}\right]} J_{\epsilon}\left(t v_{0}\right)<c^{*}$.

(ii) There are $r, \varrho>0$, such that, $J_{\epsilon}(u) \geq \varrho, \forall u \in H^{1}\left(\mathbf{R}^{N}\right)$; $\|u\|=r$.

(iii) $J_{\epsilon}\left(\gamma_{0} v_{0}\right)<0$ and $\gamma_{0} v_{0} \in B_{r}(0)^{c}$. 
Proof. Without any loss of generality we may assume that $0 \in \Omega$. Fixed $\epsilon^{*} \in$ $(0,1)$, consider $v_{0} \in C_{0}^{\infty}\left(\mathbf{R}^{N}\right)$, such that, $\int_{\mathbf{R}^{N}}\left(\left|\nabla v_{0}\right|^{2}+V_{\infty}\left|v_{0}\right|^{2}\right)=1, v_{0} \geq 0$, supp $v_{0} \subset$ $B_{R}(0) \subset \Omega$. Since $V(\epsilon x) \leq V_{\infty}$, for each $x \in B_{R}(0)$ and $\epsilon \in\left(0, \epsilon^{*}\right)$, we have $\epsilon x \in \Omega$, then, using $(8)$ and $\left(g_{2}\right)$, we get

$$
J_{\epsilon}\left(t v_{0}\right) \leq j(t)-\int_{B_{R}(0)} \int_{0}^{t v_{0}(x)} H(s-\beta) f(s) d s d x \leq j(t), \quad \text { for all } t \geq 0,
$$

where $j(t)=\frac{\theta}{2} M(0) t^{2}-\frac{t^{2^{*}}}{2^{*}} \int_{B_{R}(0)}\left|v_{0}\right|^{2^{*}}$.

Note that, the function $j$ is increasing in $\left(0, t^{*}\right)$, for some $t^{*}>0$. Since $\lim _{t \rightarrow 0} j(t)$ $=0$, there exists $\gamma_{0}>0$, independent on $\epsilon^{*}$ and $\beta$ such that,

$$
\gamma_{0}<t^{*} \text { and } \max _{t \in\left[0, \gamma_{0}\right]} J_{\epsilon}\left(t v_{0}\right) \leq j\left(\gamma_{0}\right)<c^{*}
$$

where $c^{*}$ is given in $(25)$, which prove $(i)$.

Let us prove (ii). Note that $J_{\epsilon}(0)=0$. By $\left(g_{3}\right),\left(f_{1}\right),\left(M_{1}\right)$ and choosing $\|u\|=$ $r<\gamma_{0} / 2$, there exists $\varrho>0$ such that $J_{\epsilon}(u) \geq \varrho, \forall u \in H^{1}\left(\mathbf{R}^{N}\right) ;\|u\|=r$. Moreover, using $\left(f_{3}\right)$

$$
\int_{B_{R}(0)} \int_{0}^{\gamma_{0} v_{0}(x)} H(s-\beta) f(s) d s d x \geq \int_{B_{R}(0)}\left[\gamma_{0} v_{0}-2 \beta\right] d x .
$$

By (26) we obtain

$$
J_{\epsilon}\left(\gamma_{0} v_{0}\right) \leq j\left(\gamma_{0}\right)-\int_{B_{R}(0)}\left[\gamma_{0} v_{0}+2 \beta\right] d x
$$

for $\gamma_{0}>0$ and $\beta>0$ small enough.

Theorem 3.8. Suppose that $\left(M_{1}\right),\left(f_{1}\right),\left(f_{2}\right),\left(f_{3}\right),\left(f_{4}\right),\left(V_{0}\right)$ and $\left(V_{1}\right)$ are satisfied. Then, there are $\epsilon^{*}, \beta>0$ small, such that for each $\epsilon \in\left(0, \epsilon^{*}\right)$, problem $\left(P_{\epsilon}\right)_{a}$ has a positive solution $u_{\epsilon}$ satisfying:

(i) $u_{\epsilon}$ is a weak solution of problem $\left(P_{\epsilon}\right)_{a}$ and $\left\|u_{\epsilon}\right\| \leq t_{0}, \forall \epsilon \in\left(0, \epsilon^{*}\right)$.

(ii) The set $\left\{x \in \mathbf{R}^{N}: u_{\epsilon}(x)=\beta\right\}$ has null measure, and so $u_{\epsilon}$ is a strong solution of $\left(P_{\epsilon}\right)_{a}$.

(iii) The set $\left\{x \in \mathbf{R}^{N}: u_{\epsilon}(x)>\beta\right\}$ has positive measure.

Proof. (i) Let $\beta, v_{0}$ and $\gamma_{0}$ be as in the Lemma 3.7. By Lemma 3.7, $J_{\epsilon}$ has the mountain pass geometry, it follows that there exist sequences $\left(u_{n}\right) \subset H^{1}\left(\mathbf{R}^{N}\right)$, $\left(w_{n}\right) \subset \partial J_{\epsilon}\left(u_{n}\right)$ and $\left(\xi_{n}^{*}\right) \subset \partial \Psi_{\epsilon}\left(u_{n}\right)$, such that, $w_{n}=Q_{\epsilon}^{\prime}\left(u_{n}\right)-\xi_{n}^{*}$ in $H^{-1}\left(\mathbf{R}^{N}\right)$, $\left\|w_{n}\right\|_{*}=o_{n}(1), J_{\epsilon}\left(u_{n}\right)=c_{\epsilon}+o_{n}(1)$, where $c_{\epsilon}=\inf _{\gamma \in \Gamma} \max _{t \in[0,1]} J_{\epsilon}(\gamma(t))$ and $\Gamma=\{\gamma \in$ $C\left([0,1], H^{1}\left(\mathbf{R}^{N}\right)\right): \gamma(0)=0$ and $\left.\gamma(1)=\gamma_{0} v_{0}\right\}$. Since $\left(u_{n}\right)$ is bounded in $H^{1}\left(\mathbf{R}^{N}\right)$ and $\xi_{n} \in\left[\underline{g}\left(\epsilon x, u_{n}\right), \bar{g}\left(\epsilon x, u_{n}\right)\right]$, by (12) we obtain $\left(\xi_{n}\right)$ bounded in $L^{\frac{2^{*}}{2^{*}-1}}\left(\mathbf{R}^{N}\right)$.

Now, the Proposition 3.5, implies that $u_{n} \rightarrow u_{\epsilon}$ in $H^{1}\left(\mathbf{R}^{N}\right)$ and $\xi_{n} \rightarrow \xi_{\epsilon}$ in $L^{\frac{2^{*}}{2^{*}-1}}\left(\mathbf{R}^{N}\right)$. Thus,

$$
M_{0}\left(\left\|u_{\epsilon}\right\|^{2}\right) \int_{\mathbf{R}^{N}}\left[\nabla u_{\epsilon} \nabla \varphi+V(\epsilon x) u_{\epsilon} \varphi\right]=\int_{\mathbf{R}^{N}} \xi_{\epsilon} \varphi, \quad \text { for all } \varphi \in H^{1}\left(\mathbf{R}^{N}\right)
$$

where $\xi_{\epsilon} \in\left[\underline{g}\left(\epsilon x, u_{\epsilon}\right), \bar{g}\left(\epsilon x, u_{\epsilon}\right)\right]$. By the Lemma 3.6, we get

$$
\left\|u_{\epsilon}\right\| \leq t_{0} \quad \text { and } \quad M_{0}\left(\left\|u_{\epsilon}\right\|\right)=M\left(\left\|u_{\epsilon}\right\|\right), \quad \text { for all } \epsilon \in\left(0, \epsilon^{*}\right) .
$$


Once that $\xi_{\epsilon} \in L^{\frac{2^{*}}{2^{*}-1}}\left(\mathbf{R}^{N}\right)$, from elliptic regularity theory, $u_{\epsilon} \in W^{2, \frac{2^{*}}{2^{*}-1}}\left(\mathbf{R}^{N}\right)$ and

$$
\widetilde{L}_{\epsilon}\left(u_{\epsilon}\right)(x) \in\left[\underline{g}\left(\epsilon x, u_{\epsilon}(x)\right), \bar{g}\left(\epsilon x, u_{\epsilon}(x)\right)\right] \text { a.e. in } \mathbf{R}^{N},
$$

where

$$
\widetilde{L}_{\epsilon}\left(u_{\epsilon}\right):=M\left(\left\|u_{\epsilon}\right\|^{2}\right)\left[-\Delta u_{\epsilon}+V(\epsilon x) u_{\epsilon}\right] .
$$

Taking as test function $u_{\epsilon}^{-}$, we obtain $u_{\epsilon}=u_{\epsilon}^{+} \geq 0$. By Harnack inequality, see [22, Teorema 8.20], we conclude that $u_{\epsilon}>0$. Therefore, $u_{\epsilon}$ is positive solution of $\left(P_{\epsilon}\right)_{a}$ and we prove (i).

Now, suppose that the set $\Omega_{\epsilon, \beta}:=\left\{x \in \mathbf{R}^{N}: u_{\epsilon}(x)=\beta\right\}$ has positive measure. By Stampachia Theorem, see [28], we can deduce that, $-\Delta u_{\epsilon}(x)=0$ a.e. in $\Omega_{\epsilon, \beta}$. From (29)

$$
M\left(\left\|u_{\epsilon}\right\|^{2}\right) V(\epsilon x) \beta \in[\underline{g}(\epsilon x, \beta), \bar{g}(\epsilon x, \beta)] \quad \text { a.e. in } \Omega_{\epsilon, \beta} .
$$

Once that $\beta<a$ and

$$
|g(x, t)| \leq V_{0} t / k, \quad \text { for all } x \in \mathbf{R}^{N}, t \in[0, a],
$$

thus by $(30), M(0) \leq 1 / k$, but this contradicts (10). Thus, $\left|\Omega_{\epsilon, \beta}\right|=0$, which proves (ii).

Now, suppose, by contradiction, that $\left|\left\{x \in \mathbf{R}^{N}: u_{\epsilon}(x)>\beta\right\}\right|=0$, then

$$
u_{\epsilon}(x) \leq \beta \quad \text { a.e. in } \mathbf{R}^{N},
$$

it follows from $(28),(31)$ and $(32)$, that $(M(0)-1 / k)\left\|u_{\epsilon}\right\|^{2} \leq 0$, then $u_{\epsilon}=0$, but this contradicts $J_{\epsilon}\left(u_{\epsilon}\right)=c_{\epsilon}>0$.

Lemma 3.9. Assume the same hypotheses of Theorem 3.8. Let $u_{\epsilon_{n}}$ be a positive solution of $\left(P_{\epsilon}\right)_{a}, \epsilon_{n} \rightarrow 0^{+}$and $\left(x_{n}\right) \subset \mathbf{R}^{N}$. The sequence $v_{n}(x):=u_{\epsilon_{n}}\left(x+x_{n}\right) \in$ $C\left(\mathbf{R}^{N}\right)$ and has a subsequence such that converges uniformly on compact sets to its weak limit $v \in W^{2, \frac{q}{q-1}}\left(\mathbf{R}^{N}\right) \cap C\left(\mathbf{R}^{N}\right)$.

Proof. First, we adapt for our problem some ideas found in Brezis-Kato [7], for obtain $v_{n} \in L^{p}\left(\mathbf{R}^{N}\right)$, for all $p>1$. For this end, fixed $n \in \mathbf{N}$. Using the change variable $z=x+x_{n}$, defining $v_{n}(x):=u_{\epsilon_{n}}\left(x+x_{n}\right)$ and $\xi_{n}(x):=\frac{1}{M\left(\left\|u_{\epsilon_{n}}\right\|^{2}\right)} \xi_{\epsilon_{n}}\left(x+x_{n}\right)$, we have

$$
\left\{\begin{array}{l}
-\Delta v_{n}(x)+V\left(\epsilon_{n} x+\epsilon_{n} x_{n}\right) v_{n}(x)=\xi_{n}\left(x+x_{n}\right) \text { a.e. in } \mathbf{R}^{N}, \\
v_{n} \in H^{1}\left(\mathbf{R}^{N}\right) \cap W^{2, \frac{2^{*}}{2^{*}-1}}\left(\mathbf{R}^{N}\right),
\end{array}\right.
$$

where $\xi_{\epsilon_{n}}(z) \in\left[\underline{g}\left(\epsilon_{n} z, u_{\epsilon_{n}}(z)\right), \bar{g}\left(\epsilon_{n} z, u_{\epsilon_{n}}(z)\right)\right.$ a.e. in $\mathbf{R}^{N}$.

Consider $s \geq 0, L>0, A_{n, L}=\left\{x \in \mathbf{R}^{N}:\left|v_{n}\right|^{s}<L\right\}, B_{n, L}^{c}=A_{n, L}$ and the functions

$$
v_{n, L}:=\min \left\{v_{n}\left|v_{n}\right|^{2 s}, L^{2} v_{n}\right\} \quad \text { and } \quad w_{n, L}:=\min \left\{v_{n}\left|v_{n}\right|^{s}, L v_{n}\right\} .
$$

Using the test function $v_{n, L}$, we have

$$
\int_{\mathbf{R}^{N}} \nabla v_{n} \nabla v_{n, L}+V\left(\epsilon_{n} x+\epsilon_{n} x_{n}\right) v_{n} v_{n, L}=\int_{\mathbf{R}^{N}} \xi_{n} v_{n, L}
$$

With an elementary calculation we obtain

$$
\int_{\mathbf{R}^{N}} \nabla v_{n} \nabla v_{n, L}=(2 s+1) \int_{A_{n, L}}\left|v_{n}\right|^{2 s}\left|\nabla v_{n}\right|^{2}+L^{2} \int_{B_{n, L}}\left|\nabla v_{n}\right|^{2}
$$


and

$$
\int_{\mathbf{R}^{N}}\left|\nabla w_{n, L}\right|^{2}=(s+1)^{2} \int_{A_{n, L}}\left|v_{n}\right|^{2 s}\left|\nabla v_{n}\right|^{2}+L^{2} \int_{B_{n, L}}\left|\nabla v_{n}\right|^{2} .
$$

Combining (33), (34) and (35), we find

$$
\int_{\mathbf{R}^{N}}\left|\nabla w_{n, L}\right|^{2} \leq(2 s+1) \int_{\mathbf{R}^{N}} \xi_{n} v_{n, L}
$$

From (12) there exists a constant $C>0$, independent of $L$, such that, $\left|\rho_{n}\right| \leq$ $C\left|v_{n}\right|^{2^{*}-1}$. For $0<K<L$, we obtain

$$
\begin{aligned}
& \int_{\mathbf{R}^{N}}\left|\nabla w_{n, L}\right|^{2} \leq(2 s+1) C \int_{\mathbf{R}^{N}}\left|v_{n}\right|^{2^{*}-2}\left|w_{n, L}\right|^{2} \\
& \leq(2 s+1) C K^{2^{*}-2} \int_{\left\{v_{n} \leq K\right\}}\left|v_{n}\right|^{2(s+1)}+(2 s+1) C \eta(K)\left(\int_{\left\{v_{n}>K\right\}}\left|w_{n, L}\right|^{2^{*}}\right)^{\frac{2}{2^{*}}},
\end{aligned}
$$

where $\eta(K)=\left(\int_{\left\{v_{n}>K\right\}}\left|v_{n}\right|^{2^{*}}\right)^{\frac{2^{*}-2}{2^{*}}} \rightarrow 0$ if $K \rightarrow \infty$. Thus,

$$
\int_{\mathbf{R}^{N}}\left|\nabla w_{n, L}\right|^{2} \leq(2 s+1) C K^{2^{*}-2} \int_{\mathbf{R}^{N}}\left|v_{n}\right|^{2(s+1)}+(2 s+1) C \eta(K) S \int_{\mathbf{R}^{N}}\left|\nabla w_{n, L}\right|^{2},
$$

where $S_{0}$ is the best constant in inequality

$$
\left(\int_{\mathbf{R}^{N}}|u|^{2^{*}}\right)^{\frac{2}{2^{*}}} \leq S_{0} \int_{\mathbf{R}^{N}}|\nabla u|^{2}, \quad \forall u \in D^{1,2}\left(\mathbf{R}^{N}\right) .
$$

Choosing $K$, such that, $(2 s+1) C_{1} S_{0} \eta(K) \leq \frac{1}{2}$, we have

$$
\left(\int_{\mathbf{R}^{N}}\left|w_{L, n}\right|^{2^{*}}\right)^{\frac{2}{2^{*}}} \leq 2(2 s+1) C K^{2^{*}-2} \int_{\mathbf{R}^{N}}\left|v_{n}\right|^{2(s+1)} .
$$

Since $\left|w_{n, L}\right|=\left|v_{n}\right|^{s+1}$ in $A_{n, L}$ and $\left|w_{n, L}\right| \leq\left|v_{n}\right|^{s+1}$ in $\mathbf{R}^{N}$, thus, if $v_{n} \in L^{2(s+1)}\left(\mathbf{R}^{N}\right)$, by the dominated convergence theorem, with $L \rightarrow \infty$, we obtain $v_{n} \in L^{2^{*}(s+1)}\left(\mathbf{R}^{N}\right)$.

By iteration about $s$, we have $v_{n} \in L^{p}\left(\mathbf{R}^{N}\right)$, for all $p>1$. From regularity elliptic theory, for $p$ sufficiently large, we get $v_{n} \in W^{2, p}\left(\mathbf{R}^{N}\right) \hookrightarrow C^{1, \alpha}\left(\mathbf{R}^{N}\right)$, for some $0<\alpha<1$. The boundedness of $v_{n}$ in $H^{1}\left(\mathbf{R}^{N}\right)$, see Theorem 3.8 (i), implies that $\left\|v_{n}\right\|_{C_{\text {loc }}^{1, \alpha}}$ is bounded. From the Schauder estimates, there exists $v \in C\left(\mathbf{R}^{N}\right)$, such that

$$
v_{n} \rightarrow v \text { uniformly on } K
$$

for each compact $K \subset \mathbf{R}^{N}$.

Remark 3.10. As a consequence of the proof of the above lemma we have that the solution $u_{\epsilon}$ of $\left(P_{\epsilon}\right)_{a}$ belongs to $W^{2, \frac{q}{q-1}}\left(\mathbf{R}^{N}\right) \cap C^{1, \alpha}\left(\mathbf{R}^{N}\right)$, for some $\alpha \in(0,1)$.

\section{Proof of Teorem 1.1 in the case $\left(V_{0}\right),\left(V_{1}\right)$ and $\left(V_{2}\right)$}

In this section we assume $\left(V_{0}\right),\left(V_{1}\right)$ and $\left(V_{2}\right)$,

$$
\Omega:=B_{R_{\epsilon}}(0), \quad m_{\epsilon}:=\max _{x \in \partial B_{\frac{R_{\epsilon}}{\epsilon}}(0)} u_{\epsilon}(x)>0 \quad \text { and } \quad R_{\epsilon}:=\frac{1}{\epsilon},
$$

where $u_{\epsilon}$ is a solution of the problem $\left(P_{\epsilon}\right)_{a}$ given by Theorem 3.8. 
Lemma 4.1. Suppose that $\left(M_{1}\right),\left(f_{1}\right),\left(f_{2}\right),\left(f_{3}\right),\left(f_{4}\right),\left(V_{0}\right),\left(V_{1}\right)$ and $\left(V_{2}\right)$ are satisfied. Then, given $\eta>0$, there exist $\epsilon^{*}>0$ such that

$$
m_{\epsilon}<\eta, \quad \text { for all } \epsilon \in\left(0, \epsilon^{*}\right) .
$$

Proof. Proceeding by a contradiction argument, suppose that there exists $\delta_{0}>0$, such that, for each $\eta_{n}=\frac{1}{n}$, we obtain $\epsilon_{n}>0$ small, such that,

$$
m_{\epsilon_{n}}=\max _{x \in \partial B_{\frac{R \epsilon_{n}}{\epsilon_{n}}}(0)} u_{n}(x) \geq \delta_{0}>0 \quad \text { and } \quad \epsilon_{n} \rightarrow 0,
$$

where $u_{n}:=u_{\epsilon_{n}} \in H^{1}\left(\mathbf{R}^{N}\right) \cap W^{2, \frac{q}{q-1}}\left(\mathbf{R}^{N}\right)$. By elliptic regularity theory $u_{n} \in C\left(\mathbf{R}^{N}\right)$, then, there exists $x_{n} \in \partial B_{\frac{R_{\epsilon_{n}}}{\epsilon_{n}}}(0)$, such that, $u_{n}\left(x_{n}\right)=m_{\epsilon_{n}}$. Therefore, $u_{n}\left(x_{n}\right) \geq$ $\delta_{0}>0$.

Now, note that for $v_{n}(x):=u_{n}\left(x+x_{n}\right)$ and $\varrho_{n}(x):=\xi_{n}\left(x+x_{n}\right)$,

$$
\left\{\begin{array}{l}
M\left(\left\|u_{n}\right\|^{2}\right)\left(-\Delta v_{n}(x)+V\left(\epsilon_{n} x+\epsilon_{n} x_{n}\right) v_{n}(x)\right)=\varrho_{n}(x) \text { a.e. in } \mathbf{R}^{N}, \\
v_{n} \in H^{1}\left(\mathbf{R}^{N}\right) \cap W^{2, \frac{q}{q-1}}\left(\mathbf{R}^{N}\right) .
\end{array}\right.
$$

The Theorem $3.8(\mathrm{i})$, implies that $\left(u_{n}\right)$ is bounded in $H^{1}\left(\mathbf{R}^{N}\right)$. Therefore, by (12) the sequence $\left(\xi_{n}\right) \subset\left[\underline{g}\left(\epsilon x, u_{n}\right), \bar{g}\left(\epsilon x, u_{n}\right)\right]$ is bounded in $L^{\frac{2^{*}}{2^{*}-1}}\left(\mathbf{R}^{N}\right)$.

Thus, up to a subsequence, $v_{n} \rightarrow v$ in $H^{1}\left(\mathbf{R}^{N}\right), \varrho_{n} \rightarrow \varrho$ in $L^{\frac{2^{*}}{2^{*}-1}}\left(\mathbf{R}^{N}\right), M\left(\left\|u_{n}\right\|^{2}\right)$ $\rightarrow m_{1}$ and by $\left(V_{1}\right), V\left(\epsilon_{n} x_{n}\right) \rightarrow \alpha_{1}$. Taking the limit of $n \rightarrow \infty$, let us deduce that $v$ is a weak solution solution of the problem

$$
\left\{\begin{array}{l}
m_{1}\left(-\Delta v+\alpha_{1} v\right)=\varrho \text { in } \mathbf{R}^{N}, \\
v \in H^{1}\left(\mathbf{R}^{N}\right) \cap W^{2, \frac{q}{q-1}}\left(\mathbf{R}^{N}\right) .
\end{array}\right.
$$

Using the same argument as in the proof of Lemma 3.9 we obtain $v_{n}, v \in C\left(\mathbf{R}^{N}\right)$ and a subsequence of $v_{n}$ which converges uniformly on compact sets of $\mathbf{R}^{N}$ for $v$. Then, $v(0) \geq \delta_{0}>0$ and $v \neq 0$.

Since $\left(u_{n}\right)$ is bounded in $H^{1}\left(\mathbf{R}^{N}\right), M$ is continuous with $M(t) \geq M(0)$, using similar argument as in [19, proof of the Lemma 5.1] or [1, proof of the Lemma 3.1], we obtain a sequence $\left(\epsilon_{n} x_{n}\right) \subset \mathbf{R}^{N}$, such that,

$$
\nabla V\left(\epsilon_{n} x_{n}\right) \rightarrow 0 \text { and } V\left(\epsilon_{n} x_{n}\right) \rightarrow \alpha_{1},
$$

for some $\alpha_{1} \geq V_{0}>0$. Thus, $\left(\epsilon_{n} x_{n}\right) \subset \mathbf{R}^{N}$ is a sequence $(P S)_{\alpha_{1}}$ with $\left|\epsilon_{n} x_{n}\right|=R_{\epsilon_{n}}=$ $\frac{1}{\epsilon_{n}}$. This contradicts $\left(V_{2}\right)$ and so the lemma is true.

Proof of the Theorem 1.1 for $\left(V_{0}\right),\left(V_{1}\right)$ and $\left(V_{2}\right)$. Let the constant $a$ of (9). Combining the Theorem 3.8 with the Lemma 4.1, we obtain $\epsilon^{*}>0$, such that, for each $\epsilon \in\left(0, \epsilon^{*}\right)$, the problem $\left(P_{\epsilon}\right)_{a}$ has a positive solution $u_{\epsilon}$ with $\max _{x \in \partial B_{\frac{R_{\epsilon}}{\epsilon}}(0)} u_{\epsilon}(x) \leq a$. The function $\widetilde{u}_{\epsilon}(x):=0$ in $B_{\frac{R_{\epsilon}}{\epsilon}}(0)$ and $\widetilde{u}_{\epsilon}(x):=\left(u_{\epsilon}-a\right)_{+}(x)$ in $B_{\frac{R_{\epsilon}}{\epsilon}}(0)^{c}$, belongs in $H^{1}\left(\mathbf{R}^{N}\right)$. Since

$$
M\left(\left\|u_{\epsilon}\right\|^{2}\right) \int_{\mathbf{R}^{N}}\left[\nabla u_{\epsilon} \nabla \varphi+V(\epsilon x) u_{\epsilon} \varphi\right]=\int_{\mathbf{R}^{N}} \xi_{\epsilon} \varphi, \quad \text { for all } \varphi \in H^{1}\left(\mathbf{R}^{N}\right)
$$

and $0 \leq \xi_{\epsilon} \leq V(\epsilon x) u_{\epsilon} / k$ in $B_{\frac{R_{\epsilon}}{\epsilon}}(0)^{c}$, using $\varphi=\widetilde{u}_{\epsilon}$, we have

$$
\left(M(0)-\frac{1}{k}\right) \int_{B_{\frac{R_{\epsilon}}{\epsilon}}(0)^{c}} V(\epsilon x) u_{\epsilon}\left(u_{\epsilon}-a\right)_{+} \leq 0,
$$


Existence of positive solution for Kirchhoff type problem with critical discontinuous nonlinearity 1001 thus, $u_{\epsilon}(x) \leq a$ a.e. in $B_{\frac{R_{\epsilon}}{\epsilon}}(0)^{c}$. Therefore, $u_{\epsilon}$ is a solution of (6). Moreover, we conclude the proof with the Theorem 3.8.

\section{Proof of Teorem 1.1 in the case $\left(V_{0}\right),\left(V_{1}\right)$ and $\left(V_{3}\right)$}

In this section we assume $\left(V_{0}\right),\left(V_{1}\right)$ and $\left(V_{3}\right)$,

$$
\Omega:=\Lambda \quad \text { and } \quad m_{\epsilon}:=\max _{x \in \partial \Lambda_{\epsilon}} u_{\epsilon}(x)>0,
$$

where $u_{\epsilon}$ is a solution of the problem $\left(P_{\epsilon}\right)_{a}$ given by Theorem 3.8.

Lemma 5.1. Suppose that $\left(M_{1}\right),\left(f_{1}\right),\left(f_{2}\right),\left(f_{3}\right),\left(f_{4}\right),\left(V_{0}\right),\left(V_{1}\right)$ and $\left(V_{3}\right)$ are satisfied. Thus, given $\eta>0$, there exists $\epsilon^{*}>0$, such that,

$$
m_{\epsilon}<\eta, \quad \text { for all } \epsilon \in\left(0, \epsilon^{*}\right) \text {. }
$$

Proof. Suppose that the lemma is not true. Then, repeating the arguments of proof do Lemma 4.1, we can find a sequence $\left(x_{n}\right) \subset \partial \Lambda_{\epsilon_{n}}$, such that, $\nabla V\left(\epsilon_{n} u_{n}\right) \rightarrow 0$. Once that $\left(\epsilon_{n} x_{n}\right) \in \partial \Lambda$ and $V \in C^{1}\left(\mathbf{R}^{N}, \mathbf{R}\right)$, we obtain $x_{0} \in \mathbf{R}^{N}$, such that, $x_{0} \in \partial \Lambda$ and $\nabla V\left(x_{0}\right)=0$. But this contradicts $\left(V_{3}\right)$.

Proof of the Theorem 1.1 for $\left(V_{0}\right),\left(V_{1}\right)$ and $\left(V_{3}\right)$. Using Lemma 5.1 and arguing similarly as in the proof of the case $\left(V_{0}\right),\left(V_{1}\right)$ and $\left(V_{2}\right)$ we conclude the proof of the theorem.

\section{References}

[1] Alves, C. O.: Existence of standing waves solutions for a nonlinear Schrödinger equations in $\mathbf{R}^{N}$. - J. Elliptic Parabol. Equ. 1, 2015, 231-241.

[2] Alves, C. O., A. M. Bertone, and J. V. GonçAlves: A variational approach to discontinuous problems with critical Sobolev exponents. - J. Math. Anal. App. 265, 2002, 103-127.

[3] Alves, C. O., J. A. Santos, and J. V. GonçAlves: On multiple solutions for multivalued elliptic equations under Navier boundary conditions. - J. Convex Anal. 03, 2011, 627-644.

[4] Badiale, M.: Critical exponent and discontinuous nonlinearities. - Differential Integral Equations $6,1993,1173-1185$.

[5] Badiale, M.: Some remarks on elliptic problems with discontinuous nonlinearities. - Rend. Sem. Mat. Univ. Politec. Torino 51, 1993, 331-342.

[6] Baraket, S., and G. Molica Bisci: Multiplicity results for elliptic Kirchhoff-type problems. - Adv. Nonlinear Anal. 6:1, 2017, 85-93.

[7] Brezis, H., and T. Kato: Remarks on the Schrödinger operator with singular complex potentials. - J. Math. Pures Appl. 58, 1979, 137-151.

[8] Chang, K. C.: On the multiple solutions of the elliptic differential equations with discontinuous nonlinear terms. - Sci. Sinica 21, 1978, 139-158.

[9] Chang, K.C.: The obstacle problem and partial differential equations with discontinuous nonlinearities. - Comm. Pure Appl. Math. 1978, 139-158.

[10] Chang, K. C.: Variational methods for nondifferentiable functionals and their applications to partial differential equations. - J. Math. Anal. 80, 1981, 102-129.

[11] CHEn, C.: Infinitely many solutions for $N$-Kirchhoff equation with critical exponential growth in $\mathbf{R}^{N}$. - Mediterr. J. Math. 15:1, 2018, Art. 4, 1-20.

[12] Clarke, F. H.: Optimization and nonsmooth analysis. - John Wiley \& Sons, N.Y., 1983.

[13] Clarke, F. H.: Generalized gradients and applications. - Trans. Amer. Math. Soc. 265, 1975, $247-262$. 
[14] CorrêA, F. J. S. A., and R. G. Nascimento: Existence of solutions to nonlocal elliptic equations with discontinuous terms. - Electron. J. Differential Equations 26, 2012, 1-14.

[15] Delgado, M., G. M. Figueiredo, I. Gayte, and C. Morales-Rodrigo: An optimal control problem for a Kirchhoff-type equation. - ESAIM Control Optim. Calc. Var. 23:3, 2017, $773-790$.

[16] Del Pino, M., and P. L. Felmer: Local Mountain Pass for semilinear elliptic problems in unbounded domains. - Calc. Var. Partial Differential Equations 4, 1996, 121-137.

[17] Dinu, T. L.: Standing wave solutions of Schrödinger systems with discontinuous nonlinearity in anisotropic media. - Int. J. Math. Math. Sci. 2006, 2006, 1-13.

[18] Dongdong, S., and Z. Zhitao: Uniqueness, existence and concentration of positive ground state solutions for Kirchhoff type problems in $\mathbf{R}^{3}$. - J. Math. Anal. Appl. 461:1, 2018, 128-149.

[19] Dos Santos, G. G., and G. M. Figueiredo: Existence of solutions for an NSE with discontinuous nonlinearity. - J. Fixed Point Theory, 19:1, 2017, 917-937.

[20] Figueiredo, G. M.: Existence of positive solution for a Kirchhoff problem type with critical growth via truncation argument. - J. Math. Anal. Appl. 401, 2013, 706-713.

[21] Gazzola, F., and V. Radulescu: A nonsmooth critical point theory approach to some nonlinear elliptic equations in $\mathbf{R}^{N}$. - Differential Integral Equations 13, 2000, 47-60.

[22] Gilbarg, D., and N. S. Trudinger: Elliptic partial differential equations of second order. Springer-Verlag, Berlin, 1983.

[23] Grossinho, M.R., and S. A. Tersian: An introduction to minimax theorems and their applications to differential equations. - Nonconvex Optim. Appl. 52, Springer US, 2001.

[24] Guo, J., S. MA, and G. Zhang: Solutions of the autonomous Kirchhoff type equations in $\mathbf{R}^{N}$. - Appl. Math. Lett. 82, 2018, 14-17.

[25] He, X., and X. Wu: Multiple sign-changing solutions for Kirchhoff-type equations in $\mathbf{R}^{3}$. Math. Methods Appl. Sci. 41:2, 2018, 512-524.

[26] Kirchhoff, G.: Mechanik. - Teubner, Leipzig, 1883.

[27] Lei, C., H. Suo, and C. CHU: Three solutions of a Kirchhoff type problem involving critical growth and near resonance. - Indian J. Pure Appl. Math. 49:1, 2018, 99-112.

[28] Morrey, C. B.: Multiple integrals in calculus of variations. - Springer-Verlag, Berlin, 1966.

[29] Mu, M., and H. Lu: Existence and multiplicity of positive solutions for Schrödinger-KirchhoffPoisson system with singularity. - J. Funct. Spaces 2017, Art. ID 5985962, 1-12.

[30] Radulescu, V.: Mountain pass theorems for non-differentiable functions and applications. Proc. Japan. Acad. Ser. A 69, 1993, 193-198.

[31] Shao, M., and A. Mao: Signed and sign-changing solutions of Kirchhoff type problems. - J. Fixed Point Theory Appl. 20:1, 2018, Art. 2, 1-20.

[32] She, L.-B., S. XIN, and D. YU: Multiple positive solutions for a class of Kirchhoff type equations in $\mathbf{R}^{N}$. - Bound. Value Probl. 2018, Paper No. 10, 1-13.

[33] Wu, K.: Existence of ground states for a Kirchhoff type problem without 4-superlinear condition. - Comp. Math. Appl. 75, 2018, 755-763.

[34] Wu, X., and K. TenG: Multiple non semi-trivial solutions of systems of Kirchhoff-type equations with discontinuous nonlinearities in $\mathbf{R}^{N}$. - Math. Methods Appl. Sci. 39:3, 2016, 378-393.

Received 22 April 2018 • Accepted 17 January 2019 